\title{
Light-induced stress as a primary evolutionary driver of eye origins
}

\author{
Andrew J.M. Swafford and Todd H. Oakley
}

Ecology, Evolution, and Marine Biology Department University of California Santa Barbara Santa Barbara, CA 93106

Emails: oakley@ucsb.edu; andrew.swafford@lifesci.ucsb.edu

\begin{abstract}
Eyes are quintessential complex traits and our understanding of their evolution guides models of trait evolution in general. A long-standing account of eye evolution argues natural selection favors morphological variations that allow increased functionality for sensing light (Darwin 1859; v. Salvini-Plawen and Mayr 1977; Nilsson and Pelger 1994; Nilsson 2013). While certainly true in part, this focus on visual performance does not entirely explain why diffuse photosensitivity persists even after eyes evolve, or why eyes evolved many times, each time using similar building blocks. Here we briefly review a vast literature indicating most genetic components of eyes historically responded to stress caused directly by light, including UV damage of DNA, oxidative stress, and production of aldehydes. We propose light-induced stress had a direct and prominent role in the evolution of eyes by bringing together genes to repair and prevent damage from light-stress, both before and during the evolution of eyes themselves. Stress-repair and stress-prevention genes were perhaps originally deployed as plastic responses to light and/or as beneficial mutations genetically driving expression where light was prominent. These stressresponse genes sense, shield, and refract light but only as reactions to ongoing light stress. Once under regulatory-genetic control, they could be expressed before light stress appeared, evolve as a module, and be influenced by natural selection to increase functionality for sensing light, ultimately leading to complex eyes and behaviors. Recognizing the potentially prominent role of stress in eye evolution invites discussions of plasticity and assimilation and provides a hypothesis for why similar genes are repeatedly used in convergent eyes. Broadening the drivers of eye evolution encourages consideration of multi-faceted mechanisms of plasticity/assimilation and mutation/selection for complex novelties and innovations in general.
\end{abstract}

\section{Introduction}

Understanding the evolutionary origins of complex structures and innovative functions are foundational goals of biology. Because we know a lot about their structure-function relationships and genetics, eyes serve as models for understanding complex trait evolution (Oakley and 
Speiser 2015). A commonly accepted explanation for eye evolution is that natural selection acted on variation in morphology, in turn increasing functional capabilities of photoreceptors, simple eyes, and lens-eyes (Nilsson 2009, 2013). Even though it explains many aspects of eye evolution, here we discuss how this focus on visual function is incomplete and leaves substantial features of eye evolution unexplained. Furthermore, recent work-mainly on other complex traitsincreasingly examines how multiple engines of novelty, especially plasticity, contribute to the complexity of life (West-Eberhard 2003; Moczek et al. 2011; Wagner et al. 2018, 2019). We extend causes of eye evolution beyond visual function to include stress responses as another critical driver. This focus on stress invites discussions about the relative roles of plasticity and mutation in the origins and elaboration of eyes and other complex traits.

The diversity of animal photoreception can be approximated by four rough, functional categories describing a stepwise increase in complexity of both form and function (Nilsson 2013). First, non-directional photoreceptors use only photosensitive cells, which can only measure the intensity of ambient light for use in multiple behaviors. Second, directional photoreceptors pair light-blocking pigments with photosensitive proteins. Third, low-resolution spatial vision usually uses lens-like material to begin to focus light on the retina. Finally, high-resolution spatial vision uses a lens to focus light finely and precisely onto photoreceptors. This framework hypothesizes that transitions between these morphological categories may be driven by natural selection on morphologies that allow increasingly complex behaviors (Darwin 1859; v. Salvini-Plawen and Mayr 1977; Nilsson 2013). In fact, explicit calculations of the physical and optical requirements for the different sensory tasks match well with physical capabilities inferred from the increasingly complex morphologies (Nilsson 2013). These functional categories also hold outside animals, in various single-celled organisms (Colley and Nilsson 2016; Gavelis et al. 2017; Swafford and Oakley 2018)

While the gradual elaboration of eyes may be explained by selection on visual function, it is unclear how each of these parts originated before selective pressures to refine visual acuity could shape their evolutionary trajectories (Oakley and Pankey 2008). Besides gradual modifications like deepening of pigment cups and elaboration of lenses, the complexification of eyes required discrete steps, including origins of photoreception, origins of pigmentation adjacent to photoreceptors, and origins of lens-like material in the path of light. While these discrete origins could be explained by purely random mutations that direct expression of components to evolving eyes, the randomness of this mutation-selection model does not account for some salient features of eye evolution. First, despite evolving many times separately, eyes use functionally similar, often homologous components (v. Salvini-Plawen and Mayr 1977; Picciani et al. 2018). Second, genetic components of eyes often have dual or ancestral roles in responding to stress. Third, for fitness based on visual function to be dramatically higher, morphological novelties should be paired with behavioral innovations, and it may be unlikely that compatible and purely random mutations in both behavior and vision would occur simultaneously. Here we summarize the role of stress in evolution of the components of eyes and we hypothesize that responses to stress induced by light was an instrumental force in the evolution of eyes, especially in discrete origins of lenses, eyepigments, and photoreception. 


\section{Light-Induced Stress and Eye Evolution}

\section{A. Responses to light-induced stress were critical for multiple levels of eye evolution}

Image-forming eyes evolve incredible complexity optimized for fine scale resolution, object detection, and motion tracking. However, we propose canonical visual functions need not be the only driver of eye evolution, instead eyes might arise as an emergent property of stress induced evolutionary innovation in response to a pervasive environmental toxin: light. Ultraviolet (UV) light destroys lipids, proteins, and DNA. We hypothesize that components of eyes arise, often repeatedly, from stress response networks that evolve to predict, preempt, and avoid UV damage — facilitating multiple, parallel origins of eyes. Even before the most rudimentary eyes originate, evolution may link independently evolving stress responses to create complex and effective networks to mitigate UV damage. The eventual co-option and genetic-regulatory control of these networks may lead to the origins of crucial advances in functional complexity: lenses, retinas, and pigment shields. Here, we propose a macroevolutionary history of vision in which stress responses are a primary driver of eye origins (Fig 1).

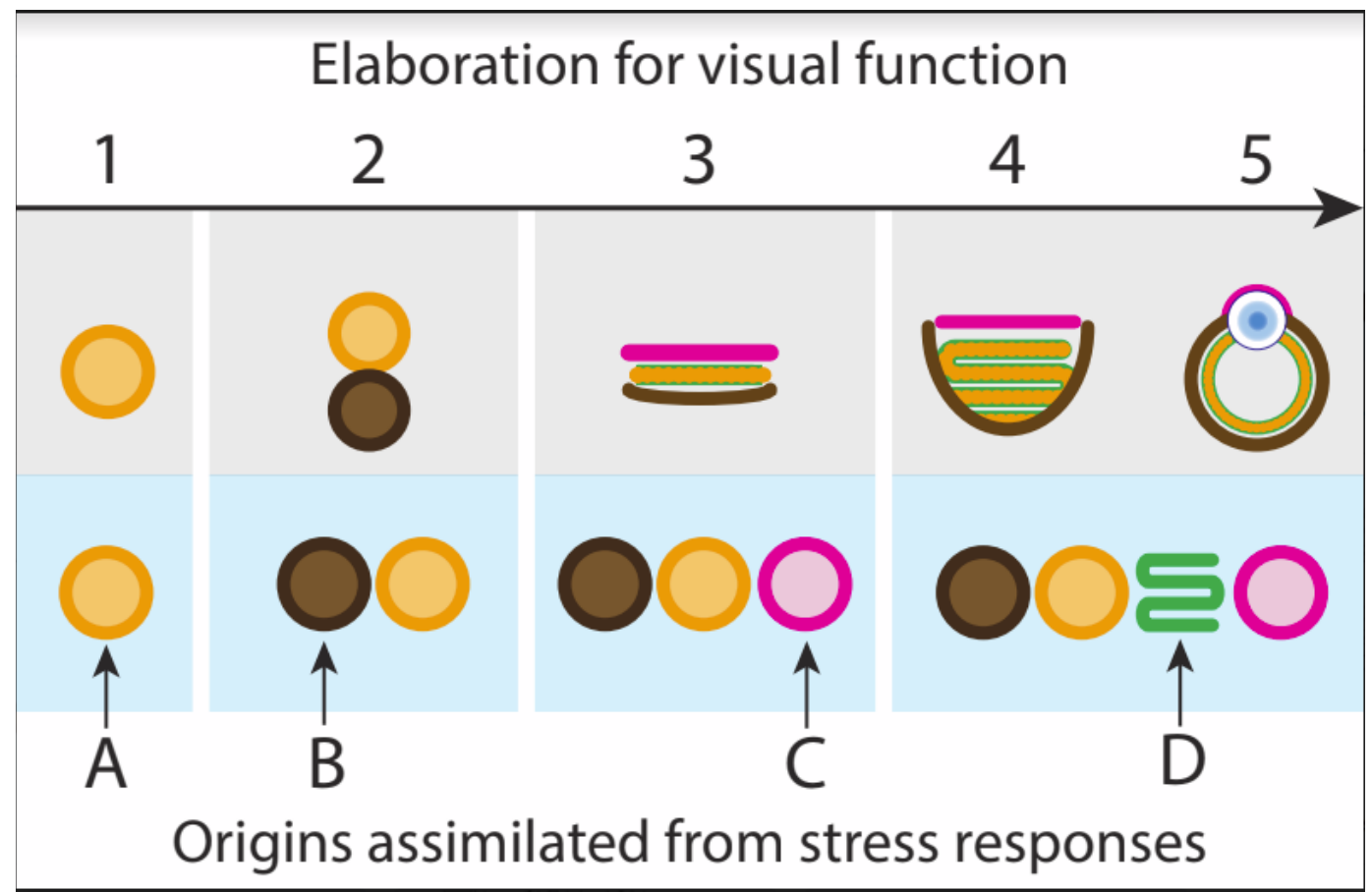

Figure 1. We propose a model of eye evolution with origins rooted in responses to light-induced stress. This builds upon previous ideas that natural selection for increased visual function is the main driver of eye evolution beginning at general photoreception (1) and ending with high-resolution image forming vision (5) (Nilsson 2013). We agree natural selection acts to elaborate visual complexity. In addition, we propose origins of parts of eyes are rooted in responses to photostress. For example $(A)$ photosensitivity may have originated to repair and predict damage to lightinduced stress, eventually leading to opsin-based photoreception. In addition, (B) pigments, such as melanin also originated to respond to light-stress. Third, $(C)$ proteins of lenses are varied and have numerous linkages to lightinduced stress. Finally, (D) cilia, which increase surface area of photoreceptive membranes, are driven by UV stress (Chavali and Gergely 2013). 
B. Response to light-stress could involve both plasticity and mutation under selection.

Phenotypic plasticity in response to the environment is one possible engine of innovation, whose contributions to complex trait evolution we are just beginning to appreciate more widely (West-Eberhard 2003; Moczek et al. 2011). Plasticity is quite different from more typical mutation driven explanations of evolution where the primary source of variation is the random mutation of genes that underlie a focal trait. In contrast, phenotypic plasticity may be a faster generator of variation within populations than mutation, creating strikingly labile systems that allow organisms to tolerate a range of environmental regimes. Unlike mutations, which are rare and often detrimental, plastic expression or development may allow immediate and appropriate responses to different environments. Over time, plastic responses may become assimilated into developmental genetic programs, which can be elaborated to create complex traits (Wagner et al. 2019). Therefore, phenotypic plasticity changes the order of events in the origins of novelty compared to mutation-selection. Under mutation-selection, random, undirected mutations create the variation in traits that selection acts upon. With phenotypic plasticity, variation first arises through plastic expression of stress/developmental programs, while mutations may occur later, allowing advantageous, yet previously plastic patterns of expression to become heritable.

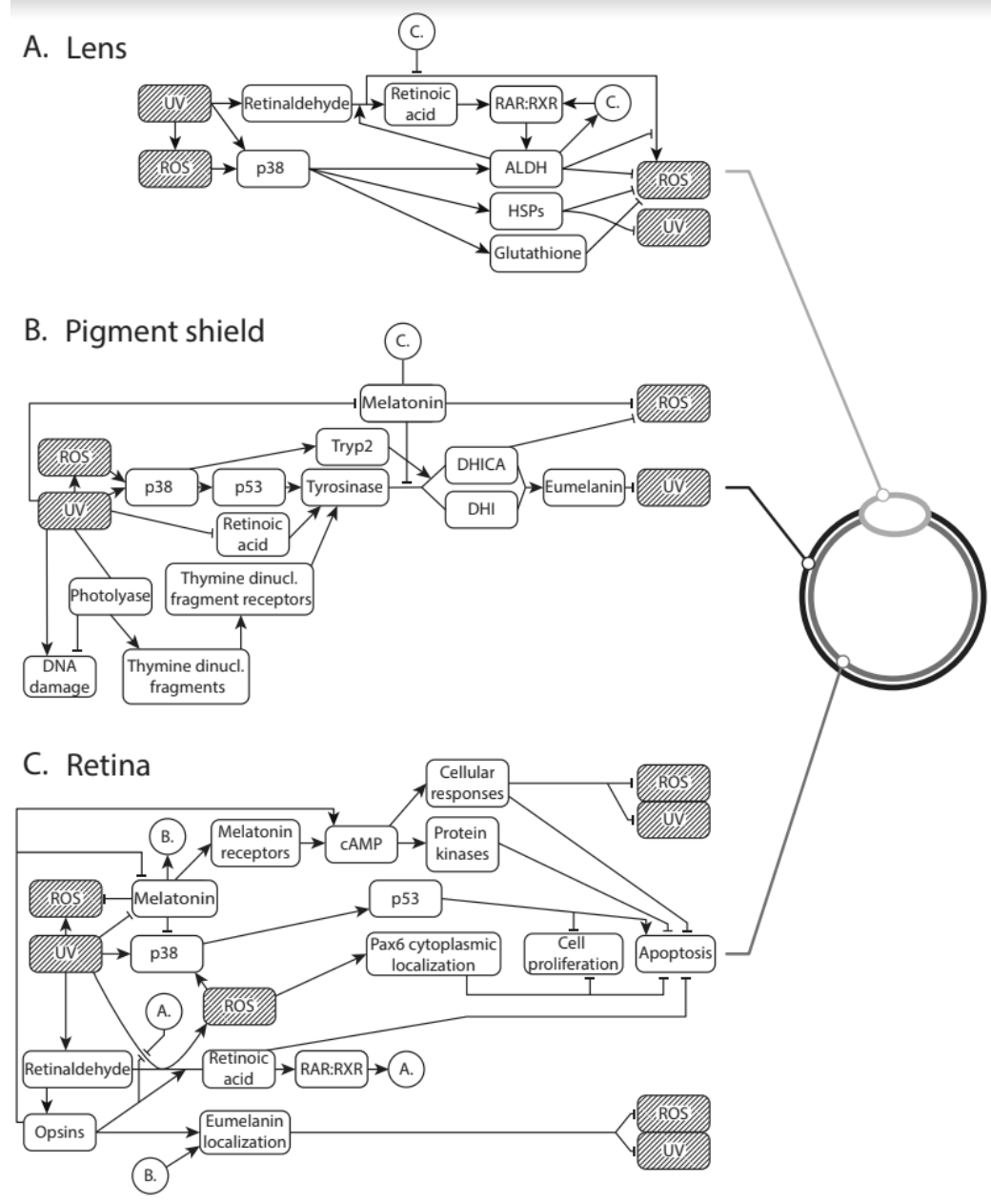

Figure 2. Diagram showing the UV- and ROS-initiated stress response pathways that underlie the origin of each functional part of vertebrate eyes. The lens (A) shows overexpression of UVblocking, chaperone, and reducing enzymes tied to the expression of light sensitive molecules in the retina (C). The pigment cup (B) is an elaboration of the original pigment shield and is a fullspectrum light blocking membrane formed from specialized portions of stress-related pathways which mitigate ROS and UV stress. The retina (C) shows overexpression of light sensitive proteins and polypeptides evolved to control pathways that rely on pathways (A) to block excess UV light and (B) to produce melanin and metabolize cytotoxic compounds in order to mitigate UV and ROS stressors. 


\section{Stress-related innovations underlie crucial components of eye evolution}

In the following sections, we discuss in more detail the role stress responses may have played in the evolution of eye components associated with discrete transitions in functional ability. Beginning with the origin of lenses, we discuss the benefits of light-protection as eyes transitioned from directional light sensors to organs capable of object resolution. We then outline a possible evolutionary path behind the molecular roots of pigments and their integration with networks governed by photosensitive proteins. We next discuss the evolution of photosensitive proteins that make up retinas, revealing another example of a stress-induced evolutionary innovation, tying stress-mitigation pathways to increasingly accurate UV sensors. Finally, we describe how these separate components might have evolved to be regulated together genetically and how this integration itself may have been related to light stress. The following sections lay out a hypothesis that addresses some gaps of acuity-driven eye evolution, showing that stress-responses to light function are also a primary driver of eye evolution.

\section{A. Lenses as Emergent Properties of Stress Mitigation}

Because lenses allow higher visual acuity, their multiple origins define crucial evolutionary transitions between directional and image-forming vision, while at the same time illustrating connections between stress and eye evolution. The genes recruited to form lenses are quite variable in origin yet very commonly are stress-response proteins. This trend has been noted for some time, but has been explained largely because of proteins' ability to remain transparent and not interfere with the visual function of the eye (Piatigorsky 2009; Nilsson 2013). However, by examining these proteins and their roles in UV-stress responses, we see possible origins of lenses by upregulation and concentration of particular proteins in front of photoreceptors to guard against toxins and mitigate UV stress. Lens proteins absorb UV light, reduce protein-protein interactions, chaperone protein folding, and metabolize cytotoxic compounds that arise as a product of both UV exposure and photoreception (Leiers et al. 2003; Lassen et al. 2008; Ou et al. 2008) (Fig. 2A). In the stepwise framework of eye evolution, the shift between directional photoreception and low resolution, image-forming vision is marked not only by the deepening of a pigment cup, but also the appearance of 'lens-like' material between the retina and the epithelial lining (Nilsson 2013). We hypothesize that the evolution of these specialized lens-like cells originates from the pressure to filter UV light from reaching an increasingly sensitive retina, which would limit external generators of ROS and aldehydes and therefore create a milder environment for photoreceptors. Thus, the stress-related origins of these crucial novelties suggest that lenses, and by extension image forming vision, originated as a protective mechanism against damage from UV and reactive oxygen species and were later elaborated by natural selection on visual function (Fig. 2A).

Lenses are constructed of stress-response proteins in multiple species (Table 1) (Piatigorsky 1998, 2009; True and Carroll 2002). As one example, the lenses of octopuses recruited several proteins into lenses, the most common of which are aldehyde dehydrogenases and glutathione S-transferases (Land 2012). The aldehyde dehydrogenases are particularly interesting because they belong to a class of proteins upregulated in response to ROS stress in animals. Aldehyde dehydrogenases aggressively find and neutralize dangerous reactive 
aldehydes such as retinaldehyde (i.e. retinal, the chromophore of opsin-based light sensitivity), 4-hydroxy-2-nonenal, and malondialdehyde, all of which are produced during UV exposure (Lassen et al. 2008). The second protein family in octopus lenses, glutathione S-transferase, detoxifies lipid molecules damaged by free radicals and ROS and acts as a free radical scavenger (Reiter 1993; Lassen et al. 2008). In mammals, lenses are comprised mostly of retinaldehyde dehydrogenase and diverse crystallin proteins. The incorporation and overexpression of aldehyde dehydrogenases and crystallins once again shows lenses are derivations of an ancestral function mitigating damage from UV stress (Piatigorsky 2009). A second protein in mammalian lenses, alpha-crystallin, arises from a larger gene family known by a different name: small heat shock proteins. These proteins, similar in function to glutathione Stransferase, work as chaperones to prevent protein-protein interactions and refold damaged proteins in response to ROS, heat, and UV stressors (de Jong et al. 1993). These properties are exceptionally useful in a lens and cornea not only because of their tolerance for environmental stressors, but also because the clarity of the lens suffers when protein-protein interactions occur.

Table 1. Common lens crystallins in vertebrates and invertebrates along with their non-lens functional classification. Table reconstructed from (Piatigorsky 1998) with additional information from (Piatigorsky et al. 2001).

\begin{tabular}{|c|c|}
\hline \multicolumn{2}{|r|}{ Lens Crystallins } \\
\hline \multicolumn{2}{|l|}{ Vertebrates } \\
\hline$\alpha$ & small heat shock proteins/chaperones; all vertebrates \\
\hline$\beta \gamma$ & members of microbial stress protein superfamily; all vertebrates \\
\hline$\varepsilon$ & lactate dehydrogenase B; ducks, crocodiles \\
\hline$\delta$ & argininosuccinate lyase; birds, reptiles \\
\hline$\tau$ & $\alpha$-enolase; turtles, ducks, other vertebrates \\
\hline$\zeta$ & novel quinone oxioreductase; guinea pig, camel, degu, llama, rock cavy \\
\hline$\mu$ & relative of bacterial ornithine cyclodeaminase; Australian marsupials \\
\hline$\eta$ & retinaldehyde dehydrogenase; elephant shrews \\
\hline$\rho$ & relative of aldo-keto reductase; frogs \\
\hline$\lambda$ & relative of hydroxyl CoA dehydrogenase; rabbits, hares \\
\hline$\pi$ & glyceraldehyde-3-phosphate dehydrogenase; geckos \\
\hline \multicolumn{2}{|l|}{ Invertebrates } \\
\hline S & glutathione S-transferase and its relatives; cephalopods \\
\hline$\Omega / L$ & relative of aldehyde dehydrogenase; cephalopods, especially octopus; scallops; squid light organ \\
\hline J & novel protein, potentially saposin/swaposin homologs; jellyfish \\
\hline Drosocrystallin & novel protein; Drosophila \\
\hline
\end{tabular}


The repeated evolution of lenses as focusing optics creates opportunities for animals to perform high-resolution spatial vision, but the advantage of improved image resolution may not be what began lens evolution each time. Photoreceptor cells of any organism are exposed to extreme amounts of UV, ROS, and heat stress in order to perform their intended function. In addition, the molecular machinery required for photoreception generates toxic aldehydes and ROS. As eyes became more sensitive by packing more photosensitive proteins into each cell, the amount of free aldehydes and the rate of ROS production would have quickly increased. In order to mitigate damage incurred by this increase in visual acuity, expression of stress response proteins would have been useful, perhaps as a protective layer inside the evolving eye that inhibits protein-protein interactions and maximizes optical clarity of the proteins. We see these proteins co-opted into lenses. Aldehyde dehydrogenases, heat shock proteins, and homologs of glutathione S-transferase are all found highly expressed in lenses. The paralogs of these genes expressed outside lenses share the same protective functions as their lens-specific counterparts, indicating stress related functions predate co-option into lenses and do not represent adaptation for the specific stresses encountered in lenses. Thus, examining the origins of genes used in lenses and high-resolution spatial vision reveal that stress may have driven co-option in response to lethal UV and ROS stress as eyes became specialized for vision. Elaboration of lenses for fine focusing and a graded refractive index in water (Sweeney et al. 2007) probably evolved later.

\section{B. Protective pigments linked to light exposure}

Shielding pigments are a crucial step in the evolution of eyes, allowing for directional photoreception by shading photoreceptors from one direction (Walne and Arnott 1967; Nilsson 2009). While pigment shields are needed for directional photoreception to evolve, the link between photosensitive proteins and antioxidant pigments may have been forged initially not for the sake of vision also, but also to protect cells from UV and ROS damage. Although animal eyes use different pigments in different taxonomic groups (Oakley and Speiser 2015), melanin is one of the best-studied light absorbing pigments recruited into eye spots, eyes, and extraocular photoreceptors. Besides its role in vision, melanin plays an integral role in protecting cells from UV damage as the end result of highly elaborate and specific protective pathways. Upon exposure to UV light, cells undergo immediate pigment darkening through the photooxidation of preexisting melanin, specifically blocking UV light and quenching ROS, while also balancing the costs of de novo melanin synthesis (Bustamante et al. 1993; Brenner and Hearing 2008; Borovansky and Riley 2011) (Fig. 2B). A closer examination of melanin production reveals intimate connections with UV stress consistent with a deep and ancient association that may predate the origins of eyes.

In vertebrates, melanin synthesis responds to the byproducts of UV-damage and stress (Fig 2b), often by regulating tyrosinase, the rate limiting enzyme in melanin synthesis. Here, we mention four different ways melanin production is connected to light, even outside of eyes. First, tyrosinase is upregulated by UV damage to DNA through a series of specific mechanisms. UV light damages DNA by causing neighboring thymines in the DNA to bond to each other. These 
damaged pieces of DNA are repaired by photolyase proteins (themselves activated by UV light), which excise and discard damaged segments, creating small fragments of DNA. The discarded fragments then upregulate tyrosinase, leading to melanin production (Eller et al. 1996). A second way tyrosinase activity is related to light is through two proteins involved in every pathway discussed in this paper (Fig. 2 A,B,C): p38 and p53. The MAPK protein p38 is activated in response to UV light and phosphorylates the inactive form of the p53 tumor suppressor protein. In addition to its many roles repairing DNA damage, controlling cell division, and regulating apoptosis of damaged cells, p53 also upregulates tyrosinase activity to create additional melanin (Khlgatian et al. 2002; Cui et al. 2007). Circadian rhythms, themselves controlled by light, further modulate p53 activity, making the interaction of p38 and p53 much more prevalent during daylight hours (Lau et al. 2014; Volonte et al. 2015). A third connection between tyrosinase and light is through the photosensitive vitamin-a derivative, retinoic acid, which plays a significant role in regulating tyrosinase activity, controlling melanin synthesis in response to retinoic acid pathways that sense light (Orlow et al. 1990; Roméro et al. 1994; Paterson et al. 2013). Finally, while the previous mechanisms all increased melanin synthesis, melatonin-a ROS and photosensitive molecule-inhibits synthesis, potentially creating a "pool" of melanin precursors during dark hours, later available for quick assembly at low metabolic costs (Logan and Weatherhead 1980) (Fig 2B). The clear links between melanin production/deployment, UV exposure, and ROS stress highlight the damage-mitigation properties that may have led to melanin's initial co-option into early photoreceptors.

\section{Origins of photoreceptors from sensors of oxidative stress}

Retinas can detect minute changes in light by densely packing photosensitive proteins into photoreceptor cells, thereby increasing sensitivity to light and allowing increased visual acuity (Nilsson 2013; Skalicky 2016). In most animal eyes, the photosensitive proteins are opsins (but see Rivera et al. 2012), usually assumed to be present in animal eyes for reasons of visual function, including quick response time and use of a chromophore that can be efficiently regenerated (Nilsson 2013). However, we suggest a driving process behind repeated co-option of opsins into retinas may also be rooted in its notable predisposition to endure and mitigate photostress. In addition to the stressors discussed earlier in this paper, UV light produces the toxic compound retinaldehyde, a necessary ligand for opsin photosensitivity (Tolleson et al. 2005). Extant opsin proteins tightly bind this free aldehyde and upregulate secondary messengers that perform a myriad of regulatory tasks in the cell (Provencio and Foster 1995; Peirson and Foster 2006; Shichida and Matsuyama 2009; Wicks et al. 2011).

The close ties between opsins and other initiators of stress response allows them to withstand the stresses of dedicated, high density photoreceptors, and could explain their cooption into each independent evolution of a retina. As eyes transition along Nilsson's (2013) stepwise evolutionary framework, the amount of opsin proteins in each photoreceptive cell retina is constantly increasing, leading to an increase in intracellular stress due to higher concentrations of retinal needed to achieve peak efficiency. Light-sensing of this caliber is a double-edged sword. The cells that are hyper-sensitized to light, and therefore the quickest to succumb to high levels of exposure (Organisciak and Vaughan 2010), are also the cells that must be exposed to the brightest light levels in order to fulfill their function. To survive these conditions, mitigation responses to light stress must be linked to immediate detection of UV 
light. Opsins perform as part of a stress response network initiated by light exposure: Nuclear retinoic acid receptors detect a drop in RA associated with UV exposure, melatonin receptors respond to the increase in ROS, and opsins detect the increase in retinaldehyde that accompanies vitamin-a metabolism/photodegradation (Tolleson et al. 2005). The speed and precision of opsin's photosensitivity may have been co-opted as a regulator for other UV stress responses, allowing immediate and comprehensive UV protection. Because of this, opsins may represent one of the only photosensitive proteins that could be expressed in high enough quantities to perform visual tasks yet also mitigate photo-stress quickly enough so photoreceptors aren't immediately killed by cytotoxic byproducts of UV exposure. Thus, we find it possible that opsin's repeated use in eyes could have initially been related to its role as a cytosolic retinaldehyde and UV light receptor, while its use as a visible light receptor is a secondary elaboration on these ancestral functions. This hypothesis predicts the ancestral function of opsins to be UV-detectors, which could be tested with comparative methods.

We suggest the light sensitivity of opsins and their subsequent use in retinas is derived from an ancestral function tracking retinaldehyde levels to coordinate pathways that mitigate stress (Fig 2C). This hypothesis is supported by three related lines of evidence. First, melatonin receptors (close relatives of opsins) track ligand concentrations as a proxy for light exposure. Melatonin acts as a powerful scavenger of ROS but it is quickly broken down by both ROS and UV light, leading to intracellular melatonin concentrations that closely track oxidative and UV stress levels (Xu et al. 2009). Melatonin receptors regulate intracellular adenylyl cyclase in response to local melatonin concentrations, and are integral in many ROS and UV damage mitigating pathways like melanin production (Fischer et al. 2008; Galano et al. 2011; Yan et al. 2018). Second, retinoic acid has clear advantages for mediating photo-stress and is used by existing photostress pathways. Retinoic acid can also be used as a sensor for UV stress, but unlike melatonin, retinoic acid fluctuations are more specifically tied to UV exposure, dropping more than fifty percent in the presence of UV light (Tolleson et al. 2005). Nuclear retinoic acid receptors use the low intracellular concentrations of retinoic acid that accompany light to stall cell division and inhibit apoptosis (Mangelsdorf et al. 1990; Konta et al. 2001; McNamara et al. 2001). Third, rhodopsin-like GPCR 161 binds retinoic acid and is tied to crucial cell processes, perhaps with functions very similar to nuclear retinoic acid receptors. GPCR-161 acts as a retinoic acid receptor controlling cell division, migration, and growth (Feigin et al. 2014; Li et al. 2015).

We hypothesize ancestral opsins also evolved as a retinoid receptor, initially regulating intracellular cyclic nucleotide levels (cAMP or cGMP) in response to retinaldehyde, the photosensitive metabolic precursor to retinoic acid (Koyanagi et al. 2008; Shichida and Matsuyama 2009). In addition, opsin's evolutionary history likely primed their downstream signaling pathways to intertwine with existing GPCR-initiated pathways for stress responses (Peirson et al. 2009; Semo et al. 2010; lyengar 2013; Bertolesi et al. 2015). Nuclear retinoic acid receptors, melatonin receptors, and opsins all play roles in directing stress responses to UV exposure, but opsins have evolved as both the most accurate photodetector and a potent activator of UV-protective pathways. Without the co-expression of each of these sensors into a coordinated ROS and UV quenching stress pathway, now found in retinas, achieving the concentration of photoreceptors needed for visual tasks could quickly kill cells through 
accumulated photooxidative stress and cytotoxic products of photodegradation (MorenoManzano et al. 1999; Organisciak et al. 1999; Kitamura et al. 2002; Du et al. 2013).

\section{Genomic regulation transforms stress response networks into a single evolutionary unit}

The concept that selection for improved visual function drives eye evolution rests on the assumption that eye morphology is heritably expressed as a module. This evolutionary step could be achieved by movement away from transient, plastic expression to coordinated developmental processes divorced from stressors and driven by transcription factors (Wagner et al. 2019). One of the largest gaps in our knowledge of eye evolution is how these regulatory relationships evolved between transcription factors and the stress response pathways outlined above (Nishina et al. 1999). In animals, UV/ROS response pathways are largely controlled by the Pax family of transcription factors, which contribute to development and patterning of lenses, pigment shields, and retinas (Ashery-Padan and Gruss 2001).

The evolution of genetic-regulatory control through Pax would have allowed disparate, plastic responses to be expressed as cohesive, permanent photosensitive modules. By examining literature surrounding a vertebrate copy of Pax, Pax-6, we hypothesize that ROS and UV stress responses may have been involved in the assimilation of transient stress pathways into predictable developmental programs (Mikkola et al. 1999; Ou et al. 2008; Laggner et al. 2017). Because of its direct role in UV-specific protection and ROS quenching (Ou et al. 2008; Laggner et al. 2017), Pax-6 would have likely co-occurred alongside the other pathways it now regulates in eye development (Fig. 1). In addition, sequence similarities between Pax-6 promoters and promoters of genes involved in crucial UV-response networks could have facilitated the co-option of Pax-6 as a 'unified' activator. Heat shock elements (found in lenses), antioxidant response elements (found throughout the eye), and p53 binding sites (cell cycle arrest and pigment expression) are all exceedingly similar to the 'optimal' Pax-6 binding site (Fig 2). As a result, very few mutations are needed to change any one of these promoter regions into one that would recognize Pax-6 as an activator (Cvekl et al. 2017). Under Pax-6 regulation, exposure to UV light would no longer be necessary, although still sufficient, to initiate the pathways discussed in sections A-D -- setting in motion the evolution of complex eyes.

\section{Summary and Conclusions}

\section{A. Stress-induced origins complete a framework of eye evolution}

Common hypotheses for the evolution of eyes rely on selection for increased visual function to explain the origins and elaboration of structures and functions of eyes. While this framework provides a reasonable explanation for the elaboration of existing traits (e.g. increasing precision of lens focusing after its origin) - it remains incomplete in its explanation of the origins of these traits. Attempting to justify the origin of eyes based solely on selection for improved visual acuity creates circular reasoning, leaving no obvious evolutionary starting point for eyes or parts of eyes to independently evolve. By focusing on eye evolution through the lens of mitigating stress from light, we suggest that the repeated co-option of genes from particular functional categories into each origin of lenses, retinas, and pigment shields could be driven by selection to mitigate photo-stress. Therefore, stress, not vision, may have often created initial 
selection to maintain co-expression and evolve co-regulation of genetic mechanisms, bringing together parts of eyes before the behavioral connections to light sensitivity could select for improved visual acuity.

\section{B. Future directions \& Acknowledging limitations}

Thinking about stress-induced origins as an engine of novelty inspires significant hypotheses that relate to processes that potentially drive complex trait evolution as a whole. Yet we acknowledge much work needs to be done to test these ideas. In constructing this framework, we face a number of restrictions that represent excellent avenues for future research. Next, we discuss several limiting factors and potential investigations that could address them, including explicit comparative work to infer the history of function and the potential confirmation bias of studies that summarize vast amounts of literature.

First, our hypotheses need to be tested with explicit comparative methods. At present, the hypotheses rely mainly on findings from basic biology and clinical research that describe protein functions in the present day. This presents two unique problems which future comparative studies would address: the timeline of stress network evolution and ancestral protein function. Without specific comparative studies, including those that take advantage of time-calibrated molecular phylogenies, it is impossible to place an order to the evolutionary events that occurred as stress networks co-opted new actors and were in turn co-opted into innovations and novelties. At the same time, with the incredible volumes of untapped sequence data, reconstructing well supported histories of genes is becoming easier. Combined, investigating the history of gene function using comparative techniques will allow testing explicit hypotheses, such as responses to stress being more ancient functions than visual functions.

A particularly compelling application of comparative techniques would be to examine the ancestral function of opsins. A critical point in the evolutionary history of animals was the ability for opsins to tightly bind retinaldehyde, forming the first bistable opsins that could be used for reliable, quick, light detection. Through experimental mutation, comparative studies, and ancestral sequence reconstruction (Chang and Donoghue 2000), we may be able to unravel how this specific function evolved. Perhaps the drive to detect smaller concentrations of retinaldehyde created a selective pressure to increase binding efficiency without the need for any form of visual system in place. Assuming the ancestral opsin was a retinaldehyde receptor, the longer each molecule of retinaldehyde remained bound, the more sensitive the cell would be to smaller and smaller concentrations of this cytotoxic compound. This pressure may have culminated in an opsin with the ability to permanently bind retinaldehyde, no longer tracking intracellular concentrations, but instead detecting the isomer of retinaldehyde trapped in the binding pocket, which changes in response to UV light. This scenario makes particular predictions, (1) that ancestors of opsin genes may lack the ability to permanently bind retinal, but still function as a retinoid receptor, and (2) that close relatives of the opsin family may have preserved their ancestral function as retinoid receptors. Testing these predictions will require a combination of additional sequencing, further comparative phylogenetic methods, and experiments examining binding properties of extant ocular and nonocular opsins.

A second concern is that because stress responses occur throughout the body, we might expect under our model of stress-induced novelty, to see eyes evolve on organisms in more broadly distributed patterns than we do. In other words, why don't eyes evolve everywhere 
an animal is exposed to light? While this concern is tempting, we point out two counter arguments. First, we are not claiming that light-stress acts alone to fully evolve eyes, instead suggesting it acts in tandem with elaboration of visual structures through natural selection. Therefore, it is logical that natural selection will affect different body regions differently, only sometimes resulting in evolution of elaborate eyes. Second, components of eyes, and sometimes even elaborated eyes, do show a broader distribution on animal bodies than complex eyes that are often only on the head. For example, chitons show a distributed system of eyes around their shells (Serb and Eernisse 2008), ciliated cells in octopus skin sense and respond to light without a nervous system (Ramirez and Oakley 2015), hydra has light sensitive neurons around the body (Plachetzki et al. 2012), sea urchins likely function as a single, large eye (Blevins and Johnsen 2004), and even human skin has melanin that might respond to light using opsins (de Assis et al. 2018). Each of these examples show that eyes or their components can evolve in many places, outlining intriguing questions about historical constraints on the evolution of eye placement (Ramirez et al. 2011). Ultimately, these examples are consistent with the hypothesis that stress responses and selection for visual acuity function in tandem as drivers of eye evolution.

A third concern that inspires future research is that our synthesis of literature from a wide array of unrelated fields risks confirmation bias. The breadth and depth of study required to unravel the molecular mechanisms of cell physiology at the level required for this paper exists mainly in a few model organisms. Because of this, our conclusions are drawn from a small subset of the tree of life, relying on examples from vertebrates and a select few invertebrates. This critique highlights the need for future work and further opens the door for future comparative studies to reinforce the support we report in a handful of well researched organisms. Interestingly, although we summarized a limited selection of pieces from the developmental network tied to eye evolution, we find that origins from light-induced stress responses likely extend far beyond our restricted presentation here (Sancar 2000; Galibert et al. 2001; Weber 2005; Piatigorsky 2009; Rivera et al. 2012; Engelen et al. 2013; Tamaru et al. 2013).

The advent of reliable single cell sequencing and advances in theory surrounding cell type evolution (Arendt et al. 2016; Wagner et al. 2019) provides another way to address issues surrounding both confirmation bias and restrictive sampling. By sequencing genes expressed in individual cells, we can examine relative enrichment of particular stress networks in particular organs. We predict cells that make up eyes will have greater representation of stress-response genes than many other cell types. Perhaps counter to this prediction, we acknowledge how shockingly common it is to find stress-related genes involved in almost any innovation or novelty (Feder and Hofmann 1999; Kültz 2005; Chavali and Gergely 2013; Wagner et al. 2018). Pleiotropic function of developmental genes and novelties tracing back to stress-responses have been superficially noted during countless studies in nearly every field, a pattern that, from an evolutionary perspective, becomes more interesting the more evidence is gathered (WestEberhard 2003; Palmer 2004; Chevin et al. 2010; Ramaswami 2014). Although the ubiquity of stress responses might make empirically demonstrating their role in eye evolution more difficult because we would lack a null model, it may nevertheless make a strong case that many traits, including eyes, evolved under stress. 
In conclusion, broadening our interpretations of the processes that drive eye evolution reveals an expanded framework that explains the origin and evolution of eyes, vision, and photoreception. Examining stress as a potential engine of plasticity and variation elucidates, once again, a surprisingly common occurrence of stress responses underlying many complex traits and developmental modules. We believe this near-ubiquitous co-option of stress genes in novelties may highlight an underappreciated macroevolutionary pattern that will illuminate, in part, the relative contributions of de novo evolution and co-option to evolution as a whole. We are excited to see future research on cell motility, sensory systems, and cell type evolution test these hypotheses and begin to delve into larger evolutionary questions about the roles of de novo evolution and co-option.

Acknowledgements

We would like to acknowledge J. Goodheart, N. Hensley, E. Ellis, and N. Picciani for their discussions and critiques of this manuscript. We would also like to acknowledge previous members of the Oakley lab: M.S. Pankey, D. Plachetzki, J. Serb, and especially D. Speiser, who contributed the seeds of these ideas in years past. We acknowledge support from NSF grant IOS-1754770.

\section{References Cited}

Arendt D, Musser JM, Baker CVH, Bergman A, Cepko C, Erwin DH, Pavlicev M, Schlosser G, Widder S, Laubichler MD, Wagner GP. 2016. The origin and evolution of cell types. Nat Rev Genet 17:744-57.

Ashery-Padan R, Gruss P. 2001. Pax6 lights-up the way for eye development. Curr Opin Cell Biol 13:706-14.

Bertolesi GE, Hehr CL, McFarlane S. 2015. Melanopsin photoreception in the eye regulates light-induced skin colour changes through the production of $\alpha-\mathrm{MSH}$ in the pituitary gland. Pigment Cell Melanoma Res 28:559-71.

Blevins E, Johnsen S. 2004. Spatial vision in the echinoid genus Echinometra. J Exp Biol 207:4249-53.

Borovansky J, Riley PA. 2011. Melanins and Melanosomes: Biosynthesis, Structure, Physiological and Pathological Functions John Wiley \& Sons.

Brenner M, Hearing VJ. 2008. The protective role of melanin against UV damage in human skin. Photochem Photobiol 84:539-49.

Bustamante J, Bredeston L, Malanga G, Mordoh J. 1993. Role of melanin as a scavenger of active oxygen species. Pigment Cell Res 6:348-53.

Chang BS, Donoghue MJ. 2000. Recreating ancestral proteins. Trends Ecol Evol 15:109-14.

Chavali PL, Gergely F. 2013. Cilia born out of shock and stress. EMBO J 32:3011-13.

Chevin L-M, Lande R, Mace GM. 2010. Adaptation, plasticity, and extinction in a changing environment: towards a predictive theory. PLoS Biol 8:e1000357.

Colley NJ, Nilsson D-E. 2016. Photoreception in Phytoplankton. Integr Comp Biol 56:764-75.

Cui R, Widlund HR, Feige E, Lin JY, Wilensky DL, Igras VE, D'Orazio J, Fung CY, Schanbacher CF, Granter SR, Fisher DE. 2007. Central role of p53 in the suntan response and pathologic hyperpigmentation. Cell 128:853-64.

Cvekl A, Zhao Y, McGreal R, Xie Q, Gu X, Zheng D. 2017. Evolutionary Origins of Pax6 Control of Crystallin Genes. Genome Biol Evol 9:2075-92.

Darwin C. 1859. On the origins of species by means of natural selection. London: Murray.

de Assis LVM, Moraes MN, Magalhães-Marques KK, Castrucci AM de L. 2018. Melanopsin and 
rhodopsin mediate UVA-induced immediate pigment darkening: Unravelling the photosensitive system of the skin. Eur J Cell Biol 97:150-62.

de Jong WW, Leunissen JA, Voorter CE. 1993. Evolution of the alpha-crystallin/small heatshock protein family. Mol Biol Evol 10:103-26.

Du Y, Veenstra A, Palczewski K, Kern TS. 2013. Photoreceptor cells are major contributors to diabetes-induced oxidative stress and local inflammation in the retina. Proc Natl Acad Sci U S A 110:16586-91.

Eller MS, Ostrom K, Gilchrest BA. 1996. DNA damage enhances melanogenesis. Proc Natl Acad Sci U S A 93:1087-92.

Engelen E, Janssens RC, Yagita K, Smits VAJ, van der Horst GTJ, Tamanini F. 2013. Mammalian TIMELESS is involved in period determination and DNA damage-dependent phase advancing of the circadian clock. PLoS One 8:e56623.

Feder ME, Hofmann GE. 1999. Heat-shock proteins, molecular chaperones, and the stress response: evolutionary and ecological physiology. Annu Rev Physiol 61:243-82.

Feigin ME, Xue B, Hammell MC, Muthuswamy SK. 2014. G-protein-coupled receptor GPR161 is overexpressed in breast cancer and is a promoter of cell proliferation and invasion. Proc Natl Acad Sci U S A 111:4191-96.

Fischer TW, Slominski A, Zmijewski MA, Reiter RJ, Paus R. 2008. Melatonin as a major skin protectant: from free radical scavenging to DNA damage repair. Exp Dermatol 17:713-30.

Galano A, Tan DX, Reiter RJ. 2011. Melatonin as a natural ally against oxidative stress: a physicochemical examination. J Pineal Res 51:1-16.

Galibert M, Carreira S, Goding CR. 2001. The Usf-1 transcription factor is a novel target for the stress-responsive p38 kinase and mediates UV - induced Tyrosinase expression. EMBO J 20:5022-31.

Gavelis GS, Keeling PJ, Leander BS. 2017. How exaptations facilitated photosensory evolution: Seeing the light by accident. Bioessays.

lyengar B. 2013. The melanocyte photosensory system in the human skin. Springerplus 2:158.

Khlgatian MK, Hadshiew IM, Asawanonda P, Yaar M, Eller MS, Fujita M, Norris DA, Gilchrest

BA. 2002. Tyrosinase gene expression is regulated by p53. J Invest Dermatol 118:126-32.

Kitamura M, Ishikawa Y, Moreno-Manzano V, Xu Q, Konta T, Lucio-Cazana J, Furusu A, Nakayama K. 2002. Intervention by retinoic acid in oxidative stress-induced apoptosis. Nephrol Dial Transplant 17 Suppl 9:84-87.

Konta T, Xu Q, Furusu A, Nakayama K, Kitamura M. 2001. Selective roles of retinoic acid receptor and retinoid $x$ receptor in the suppression of apoptosis by all-trans-retinoic acid. $J$ Biol Chem 276:12697-701.

Koyanagi M, Takano K, Tsukamoto H, Ohtsu K, Tokunaga F, Terakita A. 2008. Jellyfish vision starts with cAMP signaling mediated by opsin-G(s) cascade. Proc Natl Acad Sci U S A 105:15576-80.

Kültz D. 2005. Molecular and evolutionary basis of the cellular stress response. Annu Rev Physiol 67:225-57.

Laggner M, Pollreisz A, Schmidinger G, Schmidt-Erfurth U, Chen Y-T. 2017. Autophagy mediates cell cycle response by regulating nucleocytoplasmic transport of PAX6 in limbal stem cells under ultraviolet-A stress. PLoS One 12:e0180868.

Land MF. 2012. The evolution of lenses. Ophthalmic Physiol Opt 32:449-60.

Lassen N, Black WJ, Estey T, Vasiliou V. 2008. The role of corneal crystallins in the cellular defense mechanisms against oxidative stress. Semin Cell Dev Biol 19:100-112.

Lau AW, Liu P, Inuzuka H, Gao D. 2014. SIRT1 phosphorylation by AMP-activated protein kinase regulates p53 acetylation. Am J Cancer Res 4:245-55.

Leiers B, Kampkötter A, Grevelding CG, Link CD, Johnson TE, Henkle-Dührsen K. 2003. A stress-responsive glutathione S-transferase confers resistance to oxidative stress in Caenorhabditis elegans. Free Radic Biol Med 34:1405-15. 
Li BI, Matteson PG, Ababon MF, Nato AQ Jr, Lin Y, Nanda V, Matise TC, Millonig JH. 2015. The orphan GPCR, Gpr161, regulates the retinoic acid and canonical Wnt pathways during neurulation. Dev Biol 402:17-31.

Logan A, Weatherhead B. 1980. Post-tyrosinase inhibition of melanogenesis by melatonin in hair follicles in vitro. J Invest Dermatol 74:47-50.

Mangelsdorf DJ, Ong ES, Dyck JA, Evans RM. 1990. Nuclear receptor that identifies a novel retinoic acid response pathway. Nature 345:224-29.

McNamara P, Seo S-B, Rudic RD, Sehgal A, Chakravarti D, FitzGerald GA. 2001. Regulation of CLOCK and MOP4 by Nuclear Hormone Receptors in the Vasculature: A Humoral Mechanism to Reset a Peripheral Clock. Cell 105:877-89.

Mikkola I, Bruun JA, Bjorkoy G, Holm T, Johansen T. 1999. Phosphorylation of the transactivation domain of Pax6 by extracellular signal-regulated kinase and p38 mitogenactivated protein kinase. J Biol Chem 274:15115-26.

Moczek AP, Sultan S, Foster S, Ledón-Rettig C, Dworkin I, Nijhout HF, Abouheif E, Pfennig DW. 2011. The role of developmental plasticity in evolutionary innovation. Proc Biol Sci 278:2705-13.

Moreno-Manzano V, Ishikawa Y, Lucio-Cazana J, Kitamura M. 1999. Suppression of apoptosis by all-trans-retinoic acid. Dual intervention in the c-Jun n-terminal kinase-AP-1 pathway. J Biol Chem 274:20251-58.

Nilsson D-E. 2009. The evolution of eyes and visually guided behaviour. Philos Trans R Soc Lond B Biol Sci 364:2833-47.

Nilsson D-E. 2013. Eye evolution and its functional basis. Vis Neurosci 30:5-20.

Nilsson DE, Pelger S. 1994. A PESSIMISTIC ESTIMATE OF THE TIME REQUIRED FOR AN EYE TO EVOLVE. Proceedings of the Royal Society B-Biological Sciences 256:53-58.

Nishina S, Kohsaka S, Yamaguchi Y, Handa H, Kawakami A, Fujisawa H, Azuma N. 1999. PAX6 expression in the developing human eye. $\mathrm{Br} \mathrm{J}$ Ophthalmol 83:723-27.

Oakley TH, Pankey MS. 2008. Opening the "Black Box": The Genetic and Biochemical Basis of Eye Evolution. Evo Edu Outreach 1:390-402.

Oakley TH, Speiser DI. 2015. How Complexity Originates: The Evolution of Animal Eyes. Annu Rev Ecol Evol Syst 46:null.

Organisciak DT, Darrow RA, Barsalou L, Darrow RM, Lininger LA. 1999. Light-induced damage in the retina: differential effects of dimethylthiourea on photoreceptor survival, apoptosis and DNA oxidation. Photochem Photobiol 70:261-68.

Organisciak DT, Vaughan DK. 2010. Retinal light damage: mechanisms and protection. Prog Retin Eye Res 29:113-34.

Orlow SJ, Chakraborty AK, Pawelek JM. 1990. Retinoic acid is a potent inhibitor of inducible pigmentation in murine and hamster melanoma cell lines. J Invest Dermatol 94:461-64.

Ou J, Walczysko P, Kucerova R, Rajnicek AM, McCaig CD, Zhao M, Collinson JM. 2008. Chronic wound state exacerbated by oxidative stress in Pax6+/- aniridia-related keratopathy. J Pathol 215:421-30.

Pal K, Hwang S-H, Somatilaka B, Badgandi H, Jackson PK, DeFea K, Mukhopadhyay S. 2016. Smoothened determines $\beta$-arrestin-mediated removal of the $G$ protein-coupled receptor Gpr161 from the primary cilium. J Cell Biol 212:861-75.

Palmer AR. 2004. Symmetry breaking and the evolution of development. Science 306:828-33.

Paterson EK, Ho H, Kapadia R, Ganesan AK. 2013. 9-cis retinoic acid is the ALDH1A1 product that stimulates melanogenesis. Exp Dermatol 22:202-9.

Peirson S, Foster RG. 2006. Melanopsin: another way of signaling light. Neuron 49:331-39.

Peirson SN, Halford S, Foster RG. 2009. The evolution of irradiance detection: melanopsin and the non-visual opsins. Philos Trans R Soc Lond B Biol Sci 364:2849-65.

Piatigorsky J. 1998. Multifunctional lens crystallins and corneal enzymes. More than meets the eye. Ann N Y Acad Sci 842:7-15. 
Piatigorsky J. 2009. Gene Sharing and Evolution: The Diversity of Protein Functions Harvard University Press.

Piatigorsky J, Norman B, Dishaw LJ, Kos L, Horwitz J, Steinbach PJ, Kozmik Z. 2001. J3crystallin of the jellyfish lens: similarity to saposins. Proc Natl Acad Sci U S A 98:12362-67.

Picciani N, Kerlin JR, Sierra N, Swafford AJM, Ramirez MD, Roberts NG, Cannon JT, Daly M, Oakley TH. 2018. Prolific Origination of Eyes in Cnidaria with Co-option of Non-visual Opsins. Curr Biol 28:2413-19.e4.

Plachetzki DC, Fong CR, Oakley TH. 2012. Cnidocyte discharge is regulated by light and opsinmediated phototransduction. BMC Biol 10:17.

Provencio I, Foster RG. 1995. Circadian rhythms in mice can be regulated by photoreceptors with cone-like characteristics. Brain Res 694:183-90.

Ramaswami M. 2014. Network Plasticity in Adaptive Filtering and Behavioral Habituation. Neuron 82:1216-29.

Ramirez MD, Oakley TH. 2015. Eye-independent, light-activated chromatophore expansion (LACE) and expression of phototransduction genes in the skin of Octopus bimaculoides. $J$ Exp Biol 218:1513-20.

Ramirez MD, Speiser DI, Pankey MS, Oakley TH. 2011. Understanding the dermal light sense in the context of integrative photoreceptor cell biology. Vis Neurosci 28:265-79.

Reiter RJ. 1993. Interactions of the pineal hormone melatonin with oxygen-centered free radicals: a brief review. Braz J Med Biol Res 26:1141-55.

Rivera AS, Ozturk N, Fahey B, Plachetzki DC, Degnan BM, Sancar A, Oakley TH. 2012. Bluelight-receptive cryptochrome is expressed in a sponge eye lacking neurons and opsin. $J$ Exp Biol 215:1278-86.

Roméro C, Aberdam E, Larnier C, Ortonne JP. 1994. Retinoic acid as modulator of UVBinduced melanocyte differentiation. Involvement of the melanogenic enzymes expression. $\mathrm{J}$ Cell Sci 107 ( Pt 4):1095-1103.

Sancar A. 2000. Cryptochrome: the second photoactive pigment in the eye and its role in circadian photoreception. Annu Rev Biochem 69:31-67.

Semo M 'ayan, Gias C, Ahmado A, Sugano E, Allen AE, Lawrence JM, Tomita H, Coffey PJ, Vugler AA. 2010. Dissecting a role for melanopsin in behavioural light aversion reveals a response independent of conventional photoreception. PLoS One 5:e15009.

Serb JM, Eernisse DJ. 2008. Charting evolution's trajectory: using molluscan eye diversity to understand parallel and convergent evolution. Evolution: Education and Outreach 1:439 47.

Shichida Y, Matsuyama T. 2009. Evolution of opsins and phototransduction. Philos Trans R Soc Lond B Biol Sci 364:2881-95.

Skalicky SE. 2016. Ocular and Visual Physiology - Clinical Application Springer.

Swafford AJM, Oakley TH. 2018. Multimodal sensorimotor system in unicellular zoospores of a fungus. J Exp Biol 221.

Sweeney AM, Des Marais DL, Ban Y-EA, Johnsen S. 2007. Evolution of graded refractive index in squid lenses. J R Soc Interface 4:685-98.

Tamaru T, Hattori M, Ninomiya Y, Kawamura G, Varès G, Honda K, Mishra DP, Wang B, Benjamin I, Sassone-Corsi P, Ozawa T, Takamatsu K. 2013. ROS stress resets circadian clocks to coordinate pro-survival signals. PLoS One 8:e82006.

Tolleson WH, Cherng S-H, Xia Q, Boudreau M, Yin JJ, Wamer WG, Howard PC, Yu H, Fu PP. 2005. Photodecomposition and phototoxicity of natural retinoids. Int J Environ Res Public Health 2:147-55.

True JR, Carroll SB. 2002. Gene co-option in physiological and morphological evolution. Annu Rev Cell Dev Biol 18:53-80.

Volonte D, Zou H, Bartholomew JN, Liu Z, Morel PA, Galbiati F. 2015. Oxidative stress-induced inhibition of Sirt1 by caveolin-1 promotes p53-dependent premature senescence and 
stimulates the secretion of interleukin 6 (IL-6). J Biol Chem 290:4202-14.

v. Salvini-Plawen L, Mayr E. 1977. On the Evolution of Photoreceptors and Eyes. In: Hecht MK, Steere WC, Wallace B, editors. Evolutionary Biology Boston, MA: Springer US. p. 207-63.

Wagner GP, Erkenbrack E, Maziarz J, Chavan A. 2018. The uterine decidual stromal cell: A stabilized stress response? Journal of Reproductive Immunology.

Wagner GP, Erkenbrack EM, Love AC. 2019. Stress-Induced Evolutionary Innovation: A Mechanism for the Origin of Cell Types. .

Walne PL, Arnott HJ. 1967. The comparative ultrastructure and possible function of eyespots: Euglena granulata and Chlamydomonas eugametos. Planta 77:325-53.

Weber S. 2005. Light-driven enzymatic catalysis of DNA repair: a review of recent biophysical studies on photolyase. Biochim Biophys Acta 1707:1-23.

West-Eberhard MJ. 2003. Developmental Plasticity and Evolution Oxford University Press.

Wicks NL, Chan JW, Najera JA, Ciriello JM, Oancea E. 2011. UVA phototransduction drives early melanin synthesis in human melanocytes. Curr Biol 21:1906-11.

Xu X-R, Li X-Y, Li X-Z, Li H-B. 2009. Degradation of melatonin by UV, UV/H2O2, Fe2+/H2O2 and UV/Fe2+/H2O2 processes. Sep Purif Technol 68:261-66.

Yan G, Yu L, Jiang S, Zhu J. 2018. Melatonin antagonizes oxidative stress-induced mitochondrial dysfunction in retinal pigmented epithelium cells via melatonin receptor 1 (MT1). J Toxicol Sci 43:659-69. 